PAUL D. BOLLS

ANNIE LANG

ROBERT F. POTTER

\title{
The Effects of Message Valence and Listener Arousal on Attention, Memory, and Facial Muscular Responses to Radio Advertisements
}

This study tested the validity of using facial electromyography (EMG) as a physiological measure of the valence of radio listeners' emotional responses to advertisements and explored the effects of message valence and listener arousal on attention and memory. A within-subjects experiment was conducted in which participants listened to ten 60 -second radio advertisements that had been coded in a pretest as having either a positive or negative emotional tone. Facial EMG, heart rate, and skin conductance data were collected during exposure to the advertisements. Following exposure, participants completed free recall and recognition memory tests. Results demonstrated the validity of using facial EMG to assess the valence of emotional response to media messages. Heart rate data suggest that negative messages receive more attention than positive ones. Furthermore, how arousing a message is appears to be a better predictor of memory than message valence.

The media expose audiences to a wide range of emotional experiences. Media messages contain content explicitly designed to evoke discrete emotions such as fear, anger, joy, and sadness. Producers are aware of the intimate relationship emotion has with three primary goals of media messages: to attract attention, to entertain, and to persuade. Given the prevalence of emotionally charged media messages, it is not surprising that much attention has been directed toward investigating the relationship between emotional media content and audience members' emotional and cognitive responses to those messages (A. Lang \& Bolls, 1995; A. Lang \& Friestad, 1993; A. Lang, Newhagen, \&

COMMUNICATION RESEARCH, Vol. 28 No. 5, October 2001 627-651

(C) 2001 Sage Publications 


\section{COMMUNICATION RESEARCH • October 2001}

Reeves, 1996; Newhagen \& Reeves, 1992; Reeves, Newhagen, Maibach, Basil, \& Kurz, 1989; Thorson \& Friestad, 1989).

This study addresses three primary conceptual, methodological, and theoretical questions in understanding the relationship between emotional media messages and audience members' responses to those messages. First, this article attempts to clearly differentiate between the concepts of emotional tone (a property of the media message) and emotional response (a property of the media audience). Second, this article explores the usefulness and validity of facial electromyography (EMG) as a real-time, physiological measure of the valence of radio listeners' emotional responses to messages. Finally, this article attempts to further investigate the question of whether arousal or valence is a better predictor of memory for message content.

A major conceptual problem encountered by researchers studying emotional responses to emotional messages is the conceptualization of emotion as both a feature of media content and an audience member's response to that emotional content (A. Lang, Bolls, Potter, \& Kawahara, 1999). It is imperative to distinguish the conceptualization of emotion as a feature of the message from emotion as an audience response (elicited by the message). Media messages can be classified and described in terms of their emotional tone. Similarly, these same emotional media messages can induce the experience of emotion in audience members. The challenge facing researchers is to conceptualize clearly the difference between a message's emotional tone and an audience member's emotional experience and, at same time, to develop clear operational definitions of each.

\section{Emotion as Experience}

Considering emotion as a person's experience requires a theory of emotion. Many researchers have studied emotion and attempted to define, categorize, and better understand how people experience emotion. Two primary approaches to studying emotion dominate this research area. One is to search for primary emotions and study emotions as discrete or categorical entities. The other looks for the underlying dimensions of emotional experience. This study uses a dimensional theory of emotion. But either theoretical approach could be used to develop a conceptualization of emotional experience.

\section{A Dimensional Theory of Emotion}

A significant portion of research on emotion has used a dimensional theory of emotion (Osgood, Suci, \& Tannenbaum, 1957; P. J. Lang, Greenwald, 


\section{Bolls et al. • The Effects of Message Valence}

Bradley, \& Hamm, 1993). P. J. Lang et al. proposed a dimensional model of emotion that is founded on a biological organization of appetitive and defensive motivational connections in the human cognitive system. Lang and colleagues believed that the biological organization underlying their model accounts for much of the variance in evaluative judgments behind emotional responses. This model proposes three underlying dimensions to emotional response. The first dimension, valence, reflects the degree to which an emotional response is positive or negative. The second dimension, arousal, indicates the level of activation associated with the emotional response and ranges from very excited or energized at one extreme to very calm or sleepy at the other. A third dimension of emotional response is dominance. Measures of dominance range from a feeling of being in control during an emotional experience to a feeling of being controlled by the emotion.

Factor analyses of behavioral, self-report, and physiological data have consistently supported a strong two-factor solution consisting of arousal and valence as dimensions of emotional response (P. J. Lang et al., 1993). Dominance has been found to be a less stable dimension of emotional response and less reliably measured (Bradley \& Lang, 1994). Early in the development of a three-dimensional model of emotion, it was recognized that dominance accounts for very little of the variance in emotional response (Mehrabian \& Russell, 1974). Russell (1980) argued that dominance should maintain some theoretical importance but relegated dominance to a secondary factor. It has been proposed that dominance may be a content feature of emotional stimuli but has few motivational or behavioral consequences in determining emotional response (Bradley \& Lang, 2000). For this reason, a significant amount of research has theoretically acknowledged a three-dimensional model of emotional response but has primarily focused on manipulating and measuring arousal and valence (i.e., A. Lang, Dhillon, \& Dong, 1995; P. J. Lang et al., 1993).

The conceptualization of valence and arousal as the primary dimensions of emotional experience is quite common (Cacioppo, Petty, Losch, \& Kim, 1986; Dimberg, 1990; Hietanen, Surakka, \& Linnankoski, 1998; Jancke, Vogt, Musial, Lutz, \& Kalveram, 1996; A. Lang et al., 1995; A. Lang et al., 1996; P. J. Lang et al., 1993; Newhagen \& Reeves, 1992; Wexler, Warrenburg, Schwartz, \& Janer, 1992). This research suggests that human emotional experience can be mapped onto a two-dimensional space with valence and arousal as its axes, with a focus on emotion as it is being experienced by a person. Various techniques are used to elicit emotion in experimental participants, but the theory focuses on what happens within a person who is experiencing an emotion. 


\section{COMMUNICATION RESEARCH • October 2001}

\section{Measuring Emotional Experience}

Within this research tradition, a great deal of effort has gone into developing measures of emotional experience. According to P. J. Lang (1979), there are three types of data that can be used to measure emotional experience: behavioral, self-report, and physiological. Behavioral data include observations of facial responses and other emotional behaviors such as laughing or crying. Self-report data include verbal reports of how an emotion feels, made by the person experiencing it. Physiological data include measures of heart rate, skin conductance, and facial electromyography collected during the experience of an emotion.

As with any measure, each of these operational definitions of emotional response has inherent weaknesses. Self-report measures are subject to social response bias, particularly during the investigation of media messages covering sensitive topics. Furthermore, there is a concern that self-report measures provide measurement of responses to extreme points in message content rather than responses to the message as a whole.

Coding behavioral responses also suffers from methodological weaknesses. Researchers have recognized that it is possible for an emotional response to occur without a visible change in facial expression or behavior (Ekman, 1993; Hazlett \& Hazlett, 1999). Not only that, but the human brain is structured so that emotional response can occur before an individual engages in a behavioral response such as smiling or frowning (LeDoux, 1989). In other words, behaviors often lag behind emotional response. Also, the coding of overt behavior cannot detect preconscious emotional responses. A final drawback of behavioral observation is that fine distinctions in level of emotional response over time are difficult to observe using limited human senses.

Physiological measures can be used to assess both the arousal dimension of emotion (by measuring skin conductance) and the valence dimension of emotion (by measuring facial EMG and heart rate). However, these measures also have their drawbacks, primarily related to the data collection methods, which result in a loss of external validity (P. J. Lang et al., 1993).

Nonetheless, all three types of measures have been shown to yield valid data about how humans experience emotion. Thus, emotional experience, in this article, is conceptualized as feelings experienced by people that vary along the dimensions of arousal and valence and can be measured by obtaining behavioral, self-report, or physiological measures from the person who is experiencing the emotion.

The second task for researchers studying emotion is to differentiate emotional experience, located within human beings, from emotion as a media message feature. 
Bolls et al. • The Effects of Message Valence

\section{Emotional Tone-Emotion as a Message Feature}

What does it mean to say that a media message is emotional? Probably what is usually meant is that the message focuses on a topic about which people usually feel emotional. There is a great deal of research in media that attempts to differentiate messages in terms of their emotional content. Thorson and Friestad (1989), for example, have investigated the effectiveness of advertisements as a result of their emotional tone (positive, negative, neutral, or poignant). Research on broadcast news has focused on the emotional tone of news stories as a predictor of processing and memory (Newhagen \& Reeves, 1992; Shoemaker, 1996). A. Lang and her colleagues (1999) have investigated how messages that are more or less arousing affect online processing of the messages and memory for them.

All of these approaches assume that messages can be categorized in terms of their emotional content. That is, a message can contain emotional topics, displays of emotion, and other types of emotional information, and the degree to which a message contains this type of information can be assessed through measurement.

\section{Measuring Emotional Tone}

There are three primary methods that have been used to assess emotional tone in messages. One is to define a message as emotional if it contains displays of emotion or content that is typically considered to be emotional. This type of definition was used, for example, in studies done by Reeves and his colleagues looking at the effects of emotional media on attention and hemispheric asymmetry (Reeves et al., 1985; Reeves, A. Lang, Thorson, \& Rothchild, 1988).

A second way to measure the level of emotion in a media message is to have people rate the messages in terms of some emotional variable, such as valence, arousal, fear, disgust, and so on. When this method is used, researchers typically select messages that they believe vary in terms of emotional tone and then have groups of participants rate the messages on various emotional dimensions. It should be noted that when using this method, participants can be asked either to rate the emotional content of the message or to rate their own emotional response to the message. The resulting data are then used to indicate the emotional tone of the message.

The third primary method for evaluating emotional tone in media messages is somewhat different in that it involves varying relative emotional tone through manipulation of the message. This occurs when researchers 


\section{COMMUNICATION RESEARCH • October 2001}

wish to examine two versions of the same message that vary in terms of intensity of emotional content. In this case, a message is altered, usually through the addition of more emotional content, so that the resultant message is more emotional than the original message. For example, Newhagen and Reeves (1992) added negative emotional video to negative news stories. They assumed the addition of the negative visual material would render the later version of the news story more negative than the original version.

Thus, this article conceptualizes emotional tone as a property of the message, which can be measured through definitions of content or subject ratings. By clearly separating emotional tone from emotional experience, media researchers will be better able to investigate the relationship between the two variables without confusion.

\section{Using EMG to Measure Emotional Response}

The second goal of this article is to explore the usefulness and validity of facial EMG as a physiological measure of the valence of radio listeners' emotional responses to messages. As discussed above, emotional experience can be assessed in a variety of ways. This study was designed to test the validity of using a physiological response as an indicator of the emotional valence experienced by radio listeners during exposure. More specifically, this experiment assessed the validity of using facial EMG as a physiological measure of the valence of emotional response to radio advertisements.

The vast majority of research examining emotional responses to media has relied on self-reports of emotional experience as the primary operationalization of emotional experience. Certainly, our intent is not to diminish the contributions made by these studies. However, it is our contention that research in the area of emotional communication can be advanced by using facial EMG to provide a real-time, precise measure of how positive or negative an audience member feels while processing the message.

\section{Valence and Facial EMG}

The theoretical connection between muscle activity and emotional experience dates back to William James's (1884) peripheral theory of emotion. James proposed that the contraction of facial muscles was not only a form of emotional expression but greatly responsible for the experience of emotions themselves. Facial EMG is the modern measurement of these facial muscle contractions. This physiological method measures the electrical signal generated by the occurrence of action potentials across a group of muscles dedicated to moving particular parts of the face (Cacioppo, Tassinary, \& Fridlund, 


\section{Bolls et al. • The Effects of Message Valence}

1990). These electrical signals can be detected by the careful placement of electrodes over specific muscle groups on the surface of the skin. Facial EMG is commonly obtained from the zygomatic and corrugator muscle groups. The zygomatic muscle is located along the cheek just above the corner of the lips. Contraction of the zygomatic muscle draws the angle of the mouth backward and upward, leading the zygomatic muscle to become known as the smile muscle (Fridlund \& Izard, 1983). The corrugator muscle is located on the brow just off the bridge of the nose. Contraction of the corrugator muscle moves the brow downward and inward, associating the corrugator muscle with frowning (Fridlund \& Izard, 1983).

Facial EMG has been used extensively by psychophysiologists to measure the valence of emotional response to environmental stimuli. Fridlund and Izard (1983) did a broad review of much of the early work using this physiological measure. The valence of a wide range of visual stimuli has been shown to elicit differential responses from muscles in the face (Cacioppo et al., 1986). Much of this work used still slides of environmental stimuli that had been coded for valence of emotional tone (P. J. Lang et al., 1993). P. J. Lang and colleagues exposed participants to slides that had been rated as having positive or negative emotional tone and reported increased activity in the zygomatic (smile) muscle group for positive pictures and increased activity in the corrugator (frown) muscle group for negative pictures. Facial EMG has also been measured in response to audio tones of different intensity and valence (Dimberg, 1990), environmental sounds (Jancke et al., 1996), semantically positive and negative spoken words (Wexler, Warrenburg, Schwartz, \& Janer, 1992), and vocal affective cues (Hietanen et al., 1998). As with visual stimuli, all of these studies report increased zygomatic activity for positive stimuli and increased corrugator activity for negative stimuli.

Little research has been done to assess the validity of using facial EMG to measure emotional responses to more complex stimuli such as broadcast advertisements. Broadcast advertisements are significantly longer and contain many more emotional cues than the simple stimuli historically used by psychophysiologists. Recently, Hazlett and Hazlett (1999) compared facial EMG to self-report as a measure of emotional responses to television advertisements. Their results showed that facial EMG more effectively discriminated emotional response to specific commercials than self-reported measures. Furthermore, peaks in facial EMG response were found to be temporally related to specific emotional points in the commercial. The authors concluded that facial EMG has the potential to be a very useful tool for estimating emotional response to advertisements that vary in emotional tone.

Upon review of the literature, Hazlett and Hazlett's (1999) study appears to be the only study that assesses the validity of using facial EMG to identify 


\section{COMMUNICATION RESEARCH • October 2001}

emotional response to broadcast messages. Although their study demonstrates that facial musculature response has a temporal relationship to specific emotional cues in television advertisements, the authors did not directly assess the validity of facial EMG as a measurement of emotional valence because their stimuli were not chosen as explicit exemplars of messages with specific emotional tone. Furthermore, no studies have been done that assess facial EMG responses to radio advertisements. Therefore, this study is designed to directly assess the validity of using facial EMG to measure the valence of emotional response elicited by radio advertisements that vary in terms of the valence of their emotional tone.

Despite broadcast advertisements being longer and containing more emotional cues than still slides, there is no evidence suggesting these messages should not elicit a measurable emotional experience in listeners. Nor is there reason to expect emotional auditory messages affect the facial muscles differently than visual ones. In other words, the valence (positive or negative) of the emotional tone of a media message - in this case, a radio messageshould lead to a global increase in zygomatic (smile) muscle activity for positive messages and a global increase in corrugator (frown) muscle activity for negative messages. This leads to the first two hypotheses.

Hypothesis 1: Zygomatic muscle activity will be greater for radio advertisements with a positive emotional tone compared with radio advertisements with a negative emotional tone.

Hypothesis 2: Corrugator muscle activity will be greater for radio advertisements with a negative emotional tone compared with radio advertisements with a positive emotional tone.

\section{Message Valence and Cognitive Processing}

\section{Message Valence and Attention}

The final question addressed by this study concerns relationships among emotional experience, attention, and memory for media messages. A growing body of research has investigated the effects of message valence on attention and memory (Geiger \& Reeves, 1993; Gunter, 1987; A. Lang et al., 1995, 1996; Newhagen \& Reeves, 1992). Much of this research has been conducted under the framework of limited capacity theory (A. Lang, 2000). Limited capacity theory proposes that attention is under the dual control of the audience member and characteristics of the message. Audience members can purposefully allocate attention based on goals. Attention can also be reflexively elicited from audience members by features of the message. One way of indexing this 


\section{Bolls et al. • The Effects of Message Valence}

reflexive elicitation of attention is to measure the heart rate of the message recipient. Previous research has found that as the amount of attention being paid to a media message increases, heart rate decreases (A. Lang, 1995). In addition, this research has shown that the emotional tone of a message alters resource allocation, attention, and heart rate. It has frequently been suggested that organisms are hardwired to process negative stimuli to promote survival (Bradley, 1994; Shoemaker, 1996; Zajonc, 1984), and research investigating exposure to media messages with negative emotional tone has consistently shown decreases in heart rate associated with media messages with greater negative emotional tone (A. Lang, et al., 1995, 1996). For example, A. Lang et al. (1996) measured heart rate during television news stories that included either negative or nonnegative video footage. Results showed slower heart rate during exposure to news stories that included the presence of negative video footage compared with stories with nonnegative video footage. They suggested negative video is a production feature capable of reflexively eliciting attention to a television message. If humans are hardwired to reflexively allocate attention to negative information, then heart rate should be slower during exposure to negative stimuli regardless of whether that stimuli is primarily visual (such as with television) or exclusively auditory (such as with radio). Thus, Hypothesis 3 is as follows:

Hypothesis 3: The heart rate will be slower during exposure to radio advertisements with a negative emotional tone compared with radio advertisements with a positive emotional tone.

\section{Message Valence and Memory}

The question of whether messages with a positive or negative emotional tone are more memorable has bewildered media researchers. Researchers who have studied message valence and memory have produced a somewhat mixed set of results. Some studies suggest that negative messages are remembered better than positive messages (A. Lang \& Friestad, 1993; Newhagen \& Reeves, 1992; Reeves et al., 1989; Thorson \& Friestad, 1985). Other work suggests that positive messages are more memorable (A. Lang et al., 1995; Shapiro \& Reiger, 1989).

Cognitive psychology provides two contradictory theoretical perspectives on whether negative or positive stimuli should be more memorable. One perspective, already mentioned, is that humans are hardwired to allocate more attention to negative stimuli (Zajonc, 1984). This view has been used to argue that negative stimuli reflexively attract attention from people, resulting in

better memory for bad things. A second theory is that people have a natural 


\section{COMMUNICATION RESEARCH • October 2001}

tendency to maximize pleasantness (Fiske \& Taylor, 1984). Under this perspective, people exhibit an approach response to positive stimuli and an avoidance response to negative stimuli. Theoretically, approaching something pleasurable results in higher levels of attentional resources being allocated, and avoiding something dreadful does just the opposite.

Both of these theoretical perspectives make the assumption that increased attention allocated to a stimulus translates into better memory, which may not be true under all circumstances. A. Lang (2000) proposed that it is possible for an audience member to allocate a large amount of attentional resources toward processing a message and later have poor memory for its content. If this is the case, then valence-driven increases in attentional resources, regardless of whether they are caused by positive or negative emotional tone, may not always lead to accurate predictions about the effects of message valence on memory.

A possible explanation for conflicting theories and results concerning the effects of valence on memory could be that researchers fail to account for the effects of another dimension of emotional response, that is, arousal. Arousal, along with valence, has long been recognized as an important characteristic of a media message's emotional tone (A. Lang \& Bolls, 1995). A. Lang et al. (1995) suggested that one reason several studies have found better memory for negative media messages is that commonly, media messages with a negative emotional tone tend to be more arousing than positive ones. To test this hypothesis, they conducted an experiment whereby the emotional tone of media messages was manipulated both in terms of valence and arousal. Results showed that when arousal was controlled, positive messages were remembered slightly better than negative messages. They concluded that the arousal dimension of an emotional tone may play a larger role in determining memory than the valence dimension. However, when arousal is controlled, positive messages may be remembered better than negative messages. This leads to our final set of hypotheses:

Hypothesis 4a: If positive and negative radio advertisements result in experienced arousal levels that are not significantly different, positive messages will be better remembered than negative ones.

Hypothesis $4 b$ : If positive and negative radio advertisements result in experienced arousal levels that are significantly different, the more arousing group of messages, whether positive or negative, will be more memorable. 
Bolls et al. • The Effects of Message Valence

\section{Method}

Design

The design for this experiment was a mixed 2 (valence) $\times 5$ (message) $\times 2$ (order of presentation) repeated-measures factorial design. In this design, arousal was controlled during stimulus selection. Given the purpose of this experiment, a strong manipulation of valence rather than arousal was desired. The only between-subjects factor in this design was Order of Presentation, with two levels representing the two different orders in which the radio advertisements were presented. Participants were randomly assigned to one of the presentation orders. The two within-subjects factors were Valence, with two levels representing radio advertisements with positive or negative emotional tone, and Message, with five levels representing the five different advertisements in each level of the Valence factor. Each participant heard all 10 advertisements during the experiment.

\section{Stimulus Messages}

Stimulus messages were selected from a pool of the Mercury Awardwinning radio commercials from the years 1993 to 1995, which were provided on compact disc by the Radio Advertising Bureau. The Mercury Awards are given annually by the Radio Creative Fund to honor the best radio commercials of the calendar year. The use of award-winning advertisements served as a control for professional quality. Furthermore, because the advertisements were at least 4 years old, participants had not been recently exposed to them.To choose the stimuli for the experiment, 60 advertisements were chosen based on the researchers' evaluation of whether they had a positive or negative emotional tone. These 60 advertisements were pretested using participants $(n=17)$ similar in age to those who would participate in the experiment. The pretest procedure consisted of participants listening to the $60 \mathrm{ads}$ in one of two different orders of presentation and rating their emotional responses to each using the Self Assessment Mannequin (SAM) (P. J. Lang et al., 1993). SAM is a three-dimensional, 9-point pictorial scale that measures emotional response on the three dimensions of arousal, valence, and dominance. SAM has proven to be a reliable and valid measure of emotional response to advertisements (Morris, 1995) and emotional auditory stimuli (Potter \& Callison, 2000). Mean arousal and valence levels for each 


\section{COMMUNICATION RESEARCH • October 2001}

advertisement were calculated. Then, two groups of five ads were selectively chosen from both the extreme positive and extreme negative poles of the mean valence scores such that the groups were significantly different in their level of perceived valence, $F(1,15)=352.62, p<.001, \varepsilon^{2}=.9521$, but not significantly different on the arousal dimension $(p>.05)$. Positive messages were typified by features such as upbeat music, upbeat vocal delivery, and humor. Negative messages tended to incorporate features such as dark-sounding music, voice effects (i.e., raspy voice in an antismoking message), and fear appeal. As a repeated-measures experiment, this study used five messages at each level of Valence to assess global emotional response to Valence rather than emotional response to specific production features such as humor or fear appeal.

The 10 stimulus messages were dubbed into two orders of presentation on the audio track of a time-coded VHS videotape. One order began with a positive advertisement, the other with a negative one. Both orders then alternated between positive and negative advertisements. No two advertisements were heard sequentially in both orders. To prevent possible primacy and recency effects, no advertisement appeared exclusively in the first two or last two positions of both orders. The five positive radio advertisements consisted of ads for The Simpsons, Bud Light, Sega, Solaris Yard Basics, and Sheik condoms. The five negative radio advertisements consisted of ads for The Boston Globe, Partnership for a Drug Free America, No Guns in the Street, Abbott Northwestern Hospital, and the Massachusetts Department of Public Health. ${ }^{1}$

\section{Apparatus}

This experiment was controlled by a 386 computer using the Slimy Recognition/Reaction Time program (Newhagen, 1993) as the master controller. The stimulus tapes were played by a Panasonic videocassette recorder, with the time code being read by a Horita TRG-50 PC Time Code Reader/Generator. The time code reader interfaced with the Slimy program, which instructed the physiology computer to start and stop data collection at exact time code points corresponding to the beginning and end of each radio advertisement. The physiological data were collected and stored by a 386 computer with a LabMaster $\mathrm{AD} / \mathrm{DA}$ board installed.

Skin conductance was measured by placing two Beckman standard AG/AGCL electrodes on the participant's palmar surface after it had been wiped with distilled water to control hydration levels across participants. To help make a connection with the participant's skin, the skin conductance 


\section{Bolls et al. • The Effects of Message Valence}

electrodes were filled with an electrically neutral medium as suggested by Dawson, Schell, and Filion (1990). The signal from these electrodes was passed through a Coulbourn skin conductance coupler and was sampled 20 times per second during exposure to each message.

Heart rate and zygomatic and corrugator activity were all collected using bipolar electrode placement with a single ground electrode as reference (Marshall-Goodell, Tassinary, \& Cacioppo, 1990). Prior to electrode placement, the participant used distilled water and a clean towel to remove makeup and other substances from cheeks and above eyebrows. The experimenter prepared the participant's forearms by wiping them with distilled water and a clean towel.

A Beckman standard AG/AGCL electrode was placed on the participant's nondominant forearm to serve as the ground connection. A Beckman standard AG/AGCL electrode filled with amplification jelly was placed on each of the participant's forearms to obtain heart rate data. Heart rate was recorded as milliseconds between heart beats and later converted to beats per minute for analysis.

Zygomatic and corrugator muscle activity was recorded using two pairs of Beckman mini-AG/AGCL electrodes filled with amplification jelly. Two electrodes were placed on the skin surface of the cheek (zygomaticus major muscle), and two electrodes were placed on the skin surface along the brow (corrugator supercilii muscle). The signals from these electrodes were sampled 20 times per second during exposure to the messages. The signals were passed from participants into a bioamplifier attached to high and low pass filters and then sent through a contour follower for smoothing.

The radio advertisements were heard by the participant through the speakers of a 19-inch television set. The dimensions of the speakers were approximately 2 inches by 6 inches and were comparable to the size of the speakers in many portable stereos. Participants sat in a comfortable chair approximately 5 feet from the speakers. No video signal was sent to the set during the presentation of the radio advertisements. The videocassette

player and the experimenter were located in a separate room from the participant.

\section{Participants}

Experiment participants were 41 undergraduate students, 20 males and 21 females, recruited from telecommunication courses at a large Midwest university. All participants received course credit for their participation. 


\section{COMMUNICATION RESEARCH • October 2001}

\section{Experimental Procedures}

Participants completed the experiment individually. Upon arrival at the lab, the experimenter greeted each participant and explained that the purpose of the study was to explore how people cognitively process media messages. Participants were also informed of the procedures and small risks involved in the collection of physiological data. Informed consent was obtained from all participants.

After obtaining informed consent, the radio advertisements were played. In between each advertisement, the stimulus tape was paused to allow the participant to complete a two-page written questionnaire. The questionnaire contained a measure of attitude toward the advertisement and the SAM scale.

Following the presentation of all radio advertisements, participants completed a second experiment designed to measure responses to different styles of television news stories. This served as a distraction task for the radio experiment.

After completing the distraction task, participants completed two memory tests for the radio advertisements. The first was a free-recall measure in which participants were handed a piece of paper with two columns of answer blanks. The columns were labeled "Brand/Organization" and "Product / Issue." Participants were instructed to "write down the brand name or organization sponsoring the commercial and the specific product or issue that was advertised for as many of the radio advertisements as you can remember." Participants were verbally instructed to inform the experimenter when they were finished or when they could not remember any more commercials. No participant was allowed to spend more than 5 minutes completing the free-recall measure.

The second memory measure was a recognition test. Participants were presented with a checklist of 42 different brands and organizations. All 10 of the actual sponsors were included, with the remaining options being foils. Participants were instructed to "place an $\mathrm{X}$ on the line next to the Brand/Organization names you believe were advertised in one of the radio commercials you listened to earlier."

\section{Analysis}

All physiological data (heart rate, skin conductance, and facial EMG) were averaged over 5 -second intervals yielding 11 time points per message for analysis. Physiological data were analyzed by submitting the data to a 
Bolls et al. - The Effects of Message Valence

2 (valence) $\times 11$ (time) $\times 5$ (message) $\times 2$ (order) repeated-measures MANOVA. Time was included as a variable in the analysis because physiological response occurs across time beginning at the onset of a stimulus (Cacioppo, Tassinary, \& Berntson, 2000). Furthermore, responses to emotional media content take time to occur. A message is not immediately positive or negative, but rather, emotional responses occur as the content of the message develops over time. All memory data were scored either as 1 (hit) or 0 (miss) and submitted to a 2 (valence) $\times 5$ (message) $\times 2$ (order) repeated-measures MANOVA for analysis. The design of this experiment includes numerous effects that could be tested to control for family wise error; however, only the predicted effects were tested (Cohen \& Cohen, 1983). Only statistically significant results are reported. Order did not significantly interact with any of the predicted effects.

\section{Results}

\section{Manipulation Check}

A manipulation check was performed on perceived Valence of the radio advertisements by submitting the SAM valence scores obtained from experiment participants to a mixed 2 (valence) $\times 5$ (message) $\times 2$ (order of presentation) repeated-measures MANOVA. Results showed a successful manipulation of participants' emotional experience. The positive and negative messages were rated significantly different by experiment participants on the valence dimension of the SAM scale, $F(1,39)=209.86, p<.001$. Positive messages were rated significantly more positive $(M=6.59, S D=1.10)$ than negative messages $(M=3.15, S D=.98)$.

\section{Hypothesis 1}

This hypothesis predicted that zygomatic muscle activity would be significantly greater during positive radio advertisements compared with negative advertisements. The main effect for Valence on zygomatic muscle activity was significant, $F(1,33)=19.01, p<.001, \varepsilon^{2}=.341$. There was also a significant Valence $\times$ Time interaction, $F(1,330)=8.16, p<.001, \varepsilon^{2}=.193$. This indicates that the effect of Valence on zygomatic muscle activity is significantly different across the 60 seconds of the message. Figure 1 shows that zygomatic activity significantly increases during the 60 seconds of exposure for positive messages-presumably as the emotion builds—but is significantly lower and slightly decreases over the course of negative messages. 


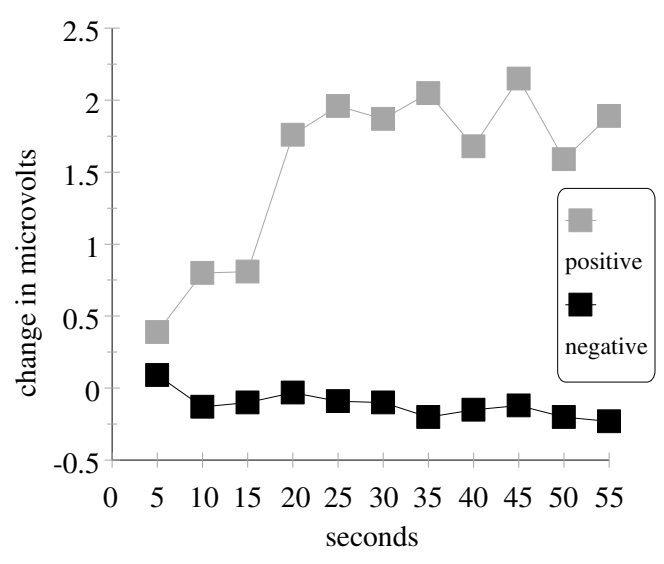

Figure 1. The Effect of Message Valence on Zygomatic (smile) Muscle Activity During Exposure to Radio Advertisements

\section{Hypothesis 2}

This hypothesis predicted that corrugator muscle activity would be greater during negative advertisements compared to positive advertisements. The main effect of Valence on corrugator muscle activity was significant, $F(1,33)=26.63, p<.001, \varepsilon^{2}=.4291$. There was also a significant Valence $\times$ Time interaction, $F(1,330)=13.83, p<.001, \varepsilon^{2}=.291$. This interaction is shown in Figure 2. Corrugator muscle activity increases and then levels off over the course of negative messages but is significantly lower and slightly decreases over the course of positive messages.

\section{Hypothesis 3}

The third hypothesis predicted that heart rate would be slower for negative radio advertisements compared to positive radio advertisements. There was a significant main effect for Valence on heart rate, $F(1,22)=5.34, p<$ $.031, \varepsilon^{2}=.158$, which is shown in Figure 3 . Heart rate was significantly slower during negative messages compared with positive messages. There was no Valence $\times$ Time interaction on heart rate.

\section{Hypothesis 4}

The final hypothesis predicted that if the positive and negative advertisements were perceived as equally arousing, then the positive radio advertise- 
Bolls et al. • The Effects of Message Valence

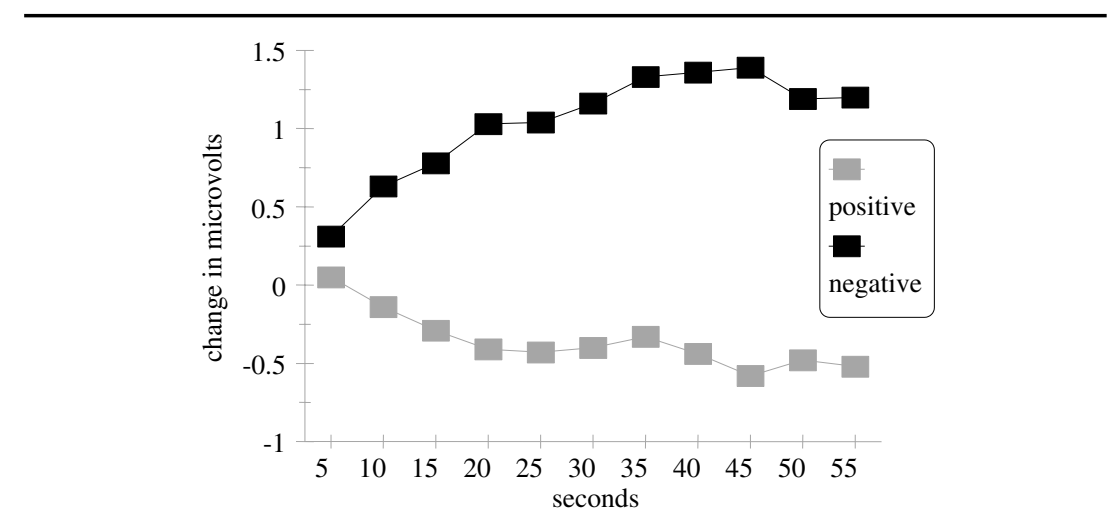

Figure 2. The Effect of Message Valence on Corrugator (frown) Muscle Activity During Exposure to Radio Advertisements

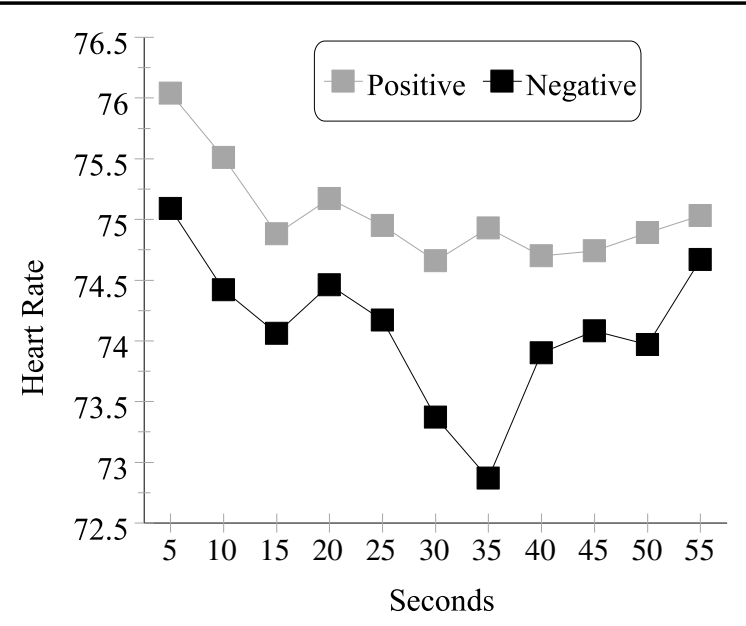

Figure 3. The Effect of Message Valence on Heart Rate During Exposure to Radio Advertisements

ments would be more memorable than the negative advertisements. However, if arousal were significantly different between the positive and negative advertisements, the more arousing group of advertisements would be more memorable regardless of valence.

The first step in testing Hypothesis 4 was to determine if the positive and negative radio advertisements used in this experiment were equally arousing to participants. ${ }^{2}$ The self-reported arousal scores from the SAM scale 


\section{COMMUNICATION RESEARCH • October 2001}

were submitted to a 2 (valence) $\times 5$ (message) $\times 2$ (order) repeated-measures MANOVA. The main effect for Valence on arousal scores was significant, $F$ (1, $39)=20.62, p<.000, \varepsilon^{2}=.333$. The positive messages $(M=6.04)$ were rated on the SAM scale as more arousing than the negative messages $(M=4.94)$. A skin conductance response analysis was also conducted. This analysis involved submitting the skin conductance data to a 2 (valence) $\times 5$ (message) $\times 2$ (order) repeated-measures MANOVA. Again, there was a main effect for Valence on the number of skin conductance responses, $F(1,34)=5.29, p<$ $.028, \varepsilon^{2}=.110$. Participants had more spontaneous skin conductance responses during positive messages $(M=3.37, S D=2.84)$ compared with negative messages $(M=2.82, S D=2.83)$. Both the SAM and skin conductance data clearly show that the participants' emotional experience was significantly more arousing during the positive advertisements compared with the negative advertisements. Because the positive radio advertisements were more arousing than the negative advertisements, the prediction for Hypothesis 4 is that the positive radio advertisements will be more memorable than the negative ones.

To test Hypothesis 4, the recognition and free recall data were submitted to a 2 (valence $) \times 5$ (message $) \times 2$ (order) repeated-measures ANOVA. There were significant main effects of Valence for both the recognition data, $F(1$, $39)=25.12, p<.001, \varepsilon^{2}=.375$, and for the free-recall data, $F(1,39)=11.68$, $p<.001, \varepsilon^{2}=.224$. Participants recognized the positive messages $(M=.90$, $S D=.14)$ better than the negative messages $(M=.71, S D=.22)$. Free recall was also better for the positive messages $(M=.70, S D=.22)$ compared with the negative messages $(M=.56, S D=.20)$. The results of this analysis are displayed in Figure 4.

\section{Discussion}

Results of this experiment demonstrate the validity of using facial EMG as a physiological measure of the valence of emotional response to radio advertisements. As predicted, activity in muscles involved in smiling and frowning clearly differentiated between messages that had been previously coded as either positive or negative in emotional tone. A comparison of Figures 1 and 2 shows zygomatic (smile) and corrugator (frown) muscles responding differentially according to message valence. During positive messages, zygomatic activity increases, whereas corrugator activity slightly decreases. During negative messages, corrugator activity increases, whereas zygomatic activity slightly decreases. Valence of the radio messages was coded using the SAM scale. Therefore, the pattern of results offers convergent validity between the SAM scale and facial EMG. Given the extensive testing 
Bolls et al. • The Effects of Message Valence

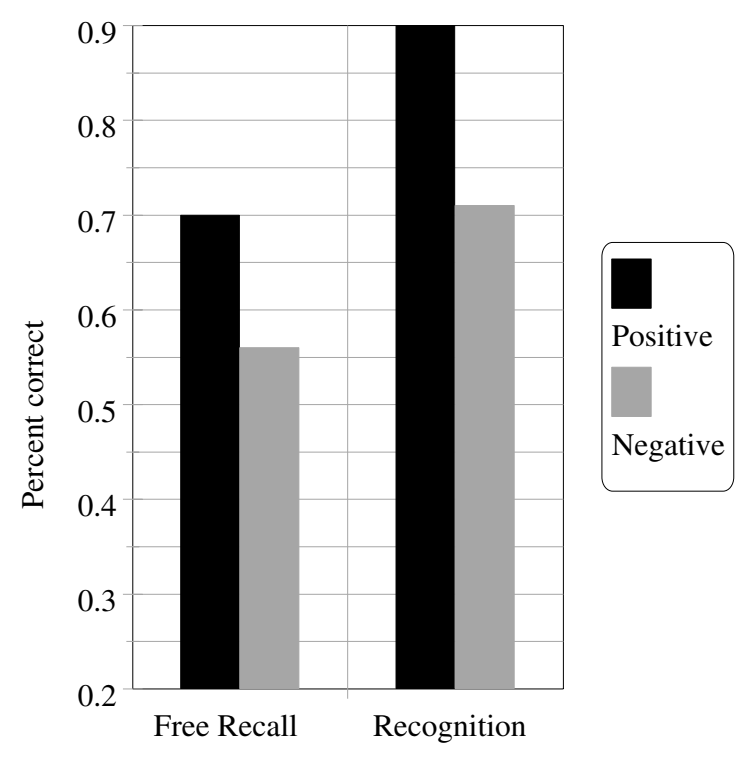

Figure 4. Free Recall and Recognition Memory Scores for the Radio Advertisements

of the SAM scale as a self-report measure of valence (A. Lang et al., 1995; P. J. Lang et al., 1993; Morris, 1995), researchers should feel fairly confident also using facial EMG as a measure of valence of emotional response. Furthermore, the pattern of results found for facial EMG activity mirror the results of previous research using facial EMG as a measure of valence during exposure to other types of stimuli. Stimuli used in this experiment were radio advertisements. However, we believe this physiological response to message valence could be robust enough to generalize across mediums. The results of this study along with work indicating the validity of using facial EMG as a measure of the valence of emotional response to still slides (P. J. Lang et al., 1993) should give researchers the confidence to use facial EMG to assess emotional response in experiments using video and computer-based stimuli. This is not to suggest other measures such as self-report and facial expression coding should be abandoned. Rather, facial EMG should be viewed as a useful addition to a media researcher's bag of tools. Facial EMG can allow researchers to assess the valence of participants' emotional response without directly asking them. This advantage may be particularly useful during the investigation of media content related to sensitive topics. Furthermore, facial EMG can identify subtle reactions to emotional media that may not be perceptible using the facial expression coding methodology. 


\section{COMMUNICATION RESEARCH • October 2001}

The measurement of facial EMG also has a level of temporal precision that could allow researchers to precisely pinpoint highly emotional media content and investigate responses to messages that are not exclusively positive or negative. Hazlett and Hazlett (1999) demonstrated that tracking facial EMG activity over the course of a television advertisement reveals emotional responses to specific content in the advertisement across time. The present study was not designed to examine emotional responses to content within a specific message; however, it is interesting to note the general trend in facial EMG activity across time even when the data is collapsed across messages. During exposure to the positive radio advertisements, zygomatic muscle activity sharply increased over the first 20 seconds of the messages and then showed both slight increases and decreases for the remaining 40 seconds. During exposure to the negative radio advertisements, corrugator activity showed a much more gradual and steady increase in activity over all 60 seconds of the messages. The differences in zygomatic and corrugator activity across time may suggest one way that cognitive processing of positive media content is different than processing of negative content. Positive content may evoke sharper and more sudden emotional responses compared to negative content. Alternatively, the difference in zygomatic and corrugator activity found in this study could be due to differences in the emotional buildup of positive versus negative radio messages. Regardless, the variation in both zygomatic and corrugator activity across time offers further evidence that facial EMG activity responds to the content of the message across time. The results of this study, taken with the work of Hazlett and Hazlett, should give researchers the confidence to use facial EMG to measure the valence of emotional response to content features occurring at specific time points in a message as well as general emotional response across messages.

There are several limitations to using facial EMG that researchers should keep in mind. Facial EMG has been noted to be susceptible to demand characteristics of the experiment (Fridlund \& Izard, 1983). Placing electrodes on the face of a participant may sensitize the participant to the fact that the experiment has something to do with facial expressions. Researchers must take special care to disguise the purpose of the experiment and distract the participant during electrode placement. The facial EMG signal is also susceptible to several sources of noise, ranging from electrical interference to movement artifact from the participant. As a result, care must also be taken to properly filter the EMG signal and to monitor any extra movements on the part of the participant. Fridlund and Cacioppo (1986) have provided a thorough discussion of the limitations and guidelines for the collection of EMG data.

The results of this experiment also support the theoretical position that people pay more attention to negative stimuli. As predicted, heart rate was 


\section{Bolls et al. • The Effects of Message Valence}

slower during exposure to negative messages compared with positive messages. Because past research shows that decelerations in heart rate indicate an increase in allocation of cognitive resources to message processing, we suggest that the current results indicate that participants allocated more attention to the negative advertisements compared with the positive ones. However, our results also show that allocating more attention to a group of messages does not always lead to better memory for message content. Even though the negative advertisements received more resource allocation, data show that the positive advertisements were more memorable. We suggest that this seeming contradiction can be explained not by the amount of attention allocated to the advertisements but rather by the levels of arousal experienced by participants during exposure. The positive advertisements in this study resulted in significantly higher levels of self-reported and physiological arousal compared with the negative ones. So even though greater attention was paid to the negative advertisements, a sense of arousal was lacking during exposure that kept the content of the advertisement from being soundly stored in long-term memory. This, in turn, prevented it from being easily retrieved during later memory tests. During exposure to the positive advertisements, however, higher arousal levels led to an increase in the amount of information being well stored-even though comparatively less was being attended to. One practical suggestion resulting from these findings is that if the goal of a media message is to be remembered, producers should put ample effort into making it arousing rather than focusing exclusively on the valence of its appeal.

One limitation to this study is that the data do not address the possible impact of dominance on the pattern of results found. As stated earlier, research has shown that dominance accounts for a very small portion of the variance in emotional response. The model of emotion used in this study is founded on deep-seeded nervous system pathways believed to be responsible for the biological expression of emotion (P. J. Lang, Bradley, \& Cuthbert, 1997). Given that valence and arousal account for most of the variance in emotional response, it is likely that these nervous system pathways, through which facial EMG response occurs, primarily respond to valence and arousal rather than dominance. Because dominance plays such a small role in emotional response, any effect of it on the results of this study is probably minimal.

One should also use care in interpreting the results based on arousal experienced during exposure to the radio messages. The results indicate that valence predicts facial EMG activity, whereas arousal predicts memory. Arousal was not manipulated the same as valence was in this study. The primary purpose of the study was to investigate how facial EMG responds to the 


\section{COMMUNICATION RESEARCH • October 2001}

valence of radio messages. Therefore, a strong manipulation of valence rather than arousal was desired. However, because arousal was measured, the data allowed for analysis of the effect of arousal on memory because experiment participants felt significantly different levels of arousal during exposure to positive versus negative messages. The hypothesis dealing with the effect of arousal on memory was based on arousal felt by the participants rather than a manipulation of arousal in the messages by the researchers.

As a laboratory experiment, this study is susceptible to several limitations. Conditions in this experiment were not meant to represent a natural listening environment. As a result, we were unable to assess the impact of other variables, such as individual goals and tasks done, in conjunction with radio listening, which would likely affect the cognitive processing of media in more natural environments. Using controlled laboratory environments to examine the cognitive and emotional variables of interest in this study allowed us to discover solid relationships before moving into the messy world of testing more ecologically valid listening environments. Furthermore, it seems likely that variables present in more natural environments may mediate the effects discovered in this experiment but would not change their direction. Therefore, despite limitations, this study makes significant contributions to the research community's ability to measure emotional responses to positive and negative media messages and deepens our understanding of the interrelationship between valence, arousal, attention, and memory.

\section{Notes}

1. Interested researchers can obtain the stimulus messages used in this experiment by contacting Paul Bolls, Edward R. Murrow School of Communication, Washington State University, P.O. Box 642520, Pullman, WA 99164-2520.

2. This initial analysis was necessary to determine if the actual participants had arousal responses to the messages consistent with the pretest participants. The original stimulus materials were chosen based on their ability to create a significant valence manipulation without a significant difference in self-reported arousal levels between levels of the Valence factor. This group of stimuli did not replicate the nonsignificant arousal findings, leaving us to test Hypothesis $4 \mathrm{~b}$.

\section{References}

Bradley, M. M. (1994). Emotional memory: A dimensional analysis. In S. Van Goozen, N. E. Van de Poll, \& J. A. Sergeant (Eds.), Emotions: Essays on emotion theory (pp. 97-134). Hillsdale, NJ: Lawrence Erlbaum. 
Bolls et al. • The Effects of Message Valence

Bradley, M. M., \& Lang, P. J. (1994). Measuring emotion: The self-assessment manikin and the semantic differential. Journal of Behavioral Therapy and Experimental Psychiatry, 25, 49-59.

Bradley, M. M., \& Lang, P. J. (2000). Measuring emotion: Behavior feeling, and physiology. In R. D. Lane \& L. Nadel (Eds.), Cognitive neuroscience of emotion. New York: Oxford University Press.

Cacioppo, J. T., Petty, R. E., Losch, M. E., \& Kim, H. S. (1986). Electromyographic activity over facial muscle regions can differentiate the valence and intensity of affective reactions. Journal of Personality and Social Psychology, 50, 260-268.

Cacioppo, J. T., Tassinary, L. G., \& Berntson, G. G. (2000). Psychophysiological science. In J. T. Cacioppo, L. G. Tassinary, \& G. G. Berntson (Eds.), Handbook of psychophysiology (pp. 3-26). New York: Cambridge University Press.

Cacioppo, J. T., Tassinary, L. G., \& Fridlund, A. J. (1990). The skeletomotor system. In J. T. Cacioppo \& L. G. Tassinary (Eds.), Principles of psychophysiology: Physical, social, and inferential elements (pp. 325-384). New York: Cambridge University Press.

Cohen, J., \& Cohen, P. (1983). Applied multiple regression/correlation analysis for the behavioral sciences. Hillsdale, NJ: Lawrence Erlbaum.

Dawson, M. E., Schell, A. M., \& Filion, D. L. (1990). The electrodermal system. In J. T. Cacioppo \& L. G. Tassinary (Eds.), Principles of psychophysiology: Physical, social, and inferential elements (pp. 295-324). New York: Cambridge University Press.

Dimberg, U. (1990). Facial electromyographic reactions and autonomic activity to auditory stimuli. Biological Psychology, 31, 137-147.

Ekman, P. (1993). Facial expression and emotion. American Psychologist, 48(4), 384-392.

Fiske, S., \& Taylor, S. (1984). Social cognition. New York: Random House.

Fridlund, A. J., \& Cacioppo, J. T. (1986). Guidelines for human electromyographic research. Psychophysiology, 23, 567-589.

Fridlund, A. J., \& Izard, C. E. (1983). Electromyographic studies of facial expressions of emotions and patterns of emotions. In J. T. Cacioppo \& R. E. Petty (Eds.), Social psychophysiology (pp. 243-286). New York: Guilford.

Geiger, S., \& Reeves, B. (1993). The effects of scene changes and semantic relatedness on attention to television. Communication Research, 20, 155-175.

Gunter, B. (1987). Poor reception: Misunderstanding and forgetting broadcast news. Hillsdale, NJ: Lawrence Erlbaum.

Hazlett, R. L., \& Hazlett, S. Y. (1999). Emotional response to television commercials: Facial EMG vs. self-report. Journal of Advertising Research, $39(2), 7-23$.

Hietanen, J. K., Surakka, V., \& Linnankoski, I. (1998). Facial electromyographic responses to vocal affect expressions. Psychophysiology, 35, 530-536.

James, W. (1884). What is an emotion? Mind, 9, 188-204. 


\section{COMMUNICATION RESEARCH • October 2001}

Jancke, L., Vogt, J., Musial, F., Lutz, K., \& Kalveram, K. T. (1996). Facial EMG responses to auditory stimuli. International Journal of Psychophysiology, $22,85-96$.

Lang, A. (1995). What can the heart tell us about thinking? In A. Lang (Ed.), Measuring psychological responses to media (pp. 99-112). Hillsdale, NJ: Lawrence Erlbaum.

Lang, A. (2000). The limited capacity model of mediated message processing. Journal of Communication, 50(1), 46-70.

Lang, A., \& Bolls, P. D. (1995). Memory for emotional television messages: Arousal, valence and capacity. Paper presented at the annual conference of the International Communication Association, Albuquerque, NM.

Lang, A., Bolls, P. D., Potter, R. F., \& Kawahara, K. (1999). The effects of production pacing and arousing content on the information processing of television messages. Journal of Broadcasting and Electronic Media, 43(4), 451-475.

Lang, A., Dhillon, K., \& Dong, Q. (1995). The effects of emotional arousal and valence on television viewers' cognitive capacity and memory. Journal of Broadcasting and Electronic Media, 39, 313-327.

Lang, A., \& Friestad, M. (1993). Emotion, hemispheric specialization and visual and verbal memory for television messages. Communication Research, 20, 647-670.

Lang, A., Newhagen, J. \& Reeves, B. (1996). Negative video as structure: Emotion, attention, capacity, and memory. Journal of Broadcasting and Electronic Media, 40, 460-477.

Lang, P. J. (1979). A bio-informational theory of emotional imagery. Psychophysiology, 16(6), 495-512.

Lang, P. J., Bradley, M. M., \& Cuthbert, M. M. (1997). Motivated attention: Affect, activation and action. In P. J. Lang, R. F. Simons, \& M. T. Balaban (Eds.), Attention and orienting: Sensory and motivational processes (pp. 97-136). Hillsdale, NJ: Lawrence Erlbaum.

Lang, P. J., Greenwald, M., Bradley, M. M., \& Hamm, A. O. (1993). Looking at pictures: Evaluative, facial, visceral, and behavioral responses. Psychophysiology, 30, 261-273.

LeDoux, J. E. (1989). Cognitive-emotional interactions in the brain. Cognition and Emotion, 3(4), 267-289.

Marshall-Goodell, B. S., Tassinary, L. G., \& Cacioppo, J. T. (1990). Principles of bioelectrical measurement. In J. T. Cacioppo \& L. G. Tassinary (Eds.), Principles of psychophysiology: Physical, social, and inferential elements (pp. 113-148). New York: Cambridge University Press.

Mehrabian, A., \& Russell, J. A. (1974). An approach to environmental psychology. Cambridge, MA: MIT.

Morris, J. D. (1995). Observations: SAM the self assessment mannequin-An efficient cross-cultural measurement of emotional response. Journal of Advertising Research, 35(6), 63-68.

Newhagen, J. (1993). Psychological instrumentation of memory and yes-no recognition. College Park, MA: University of Maryland. 
Bolls et al. • The Effects of Message Valence

Newhagen, J., \& Reeves, B. (1992). This evening's bad news: Effects of compelling negative television news images on memory. Journal of Communication, 42, 25-41.

Osgood, C., Suci, G., \& Tannenbaum, P. (1957). The measurement of meaning. Urbana: University of Illinois.

Potter, R. F., \& Callison, C. (2000). Sounds exciting! The effects of audio complexity on listeners' attitudes and memory for radio promotional announcements. Journal of Radio Studies, 7, 29-51.

Reeves, B., Lang, A., Thorson, E., \& Rothschild, M. (1988). Hemispheric lateralization and the processing of emotional television scenes. Human Communication Research, 15, 493-508.

Reeves, B., Newhagen, J., Maibach, E., Basil, M., \& Kurz, K. (1989). Negative and positive television messages: Effects of message type and message context on attention and memory. American Behavioral Scientist, 34, 679-694.

Reeves, B., Thorson, E., Rothschild, M., McDonald, D., Hirsch, J., \& Goldstein, R. (1985). Attention to television: Intra stimulus effects of movement and scene changes on alpha variation over time. International Journal of Neuroscience, $25,241-255$.

Russell, J. A. (1980). A circumplex model of affect. Journal of Personality and Social Psychology, 36, 1161-1178.

Shapiro, M., \& Rieger, R. (1989). Comparing positive and negative political advertising. Paper presented at the annual conference of the International Communication Association, San Francisco.

Shoemaker, P. J. (1996). Hardwired for news: Using biological and cultural evolution to explain the surveillance function. Journal of Communication, 46(3), 32-47.

Thorson, E., \& Friestad, M. (1989). The effects of emotion on episodic memory for television commercials. In A. Tybout \& P. Cafferata (Eds.), Advertising and consumer psychology (pp. 305-325). New York: Lexington Press.

Wexler, B. E., Warrenburg, S., Schwartz, G. E., \& Janer, L. D. (1992). EEG and EMG repsonses to emotion-evoking stimuli processed without conscious awareness. Neuropsychologia, 30, 1065-1079.

Zajonc, R. B. (1984). The interaction of affect and cognition. In K. R. Scherer \& P. Ekman (Eds.), Approaches to emotion (pp. 239-246). Hillsdale, NJ: Lawrence Erlbaum. 